\section{Lending a hand to protective immunity}

Rudensky and colleagues have revealed an unexpected role for regulatory $\mathrm{T}\left(\mathrm{T}_{\mathrm{Reg}}\right)$ cells during early infection. Rather than hindering the establishment of protective immunity during infection as has often been anticipated, these authors propose instead that $\mathrm{T}_{\text {Reg }}$ cells facilitate early protective immune responses by allowing a timely entry of immune cells to infected tissues.

$\mathrm{T}_{\text {Reg }}$ cells are key players in the immunes system's self-check mechanism, which ensures maintenance of immune system homeostasis by suppressing late-stage immune responses that have successfully tackled invading pathogens, and which prevents the development of autoimmunity. But defining the role of these cells during the early stages of infection, when the suppression of immune responses is undesirable, has challenged immunologists for some time, with several studies proposing mutually exclusive scenarios. Now, in an attempt to clarify this issue, Rudensky and colleagues have examined the role of $\mathrm{T}_{\text {Reg }}$ cells during mucosal herpes simplex virus (HSV) infection.

Using FOXP3 ${ }^{\text {GFP }}$ knock-in mice (which harbour $\mathrm{T}_{\text {Reg }}$ cells that express green fluorescent protein) the authors found that $\mathrm{T}_{\mathrm{Reg}}$ cells and 'effector' cells (or FOXP3- T cells) respond to HSV infection in an almost identical manner, ruling out the possibility that $\mathrm{T}_{\mathrm{Reg}}$ cells remain refractory during early infection. Having established that local viral infection activates $\mathrm{T}_{\mathrm{Reg}}$ cells, the next challenge was to determine what their specific role in the immune response might be. To address this, the authors used FOXP $3^{\text {DTR }}$ knock-in

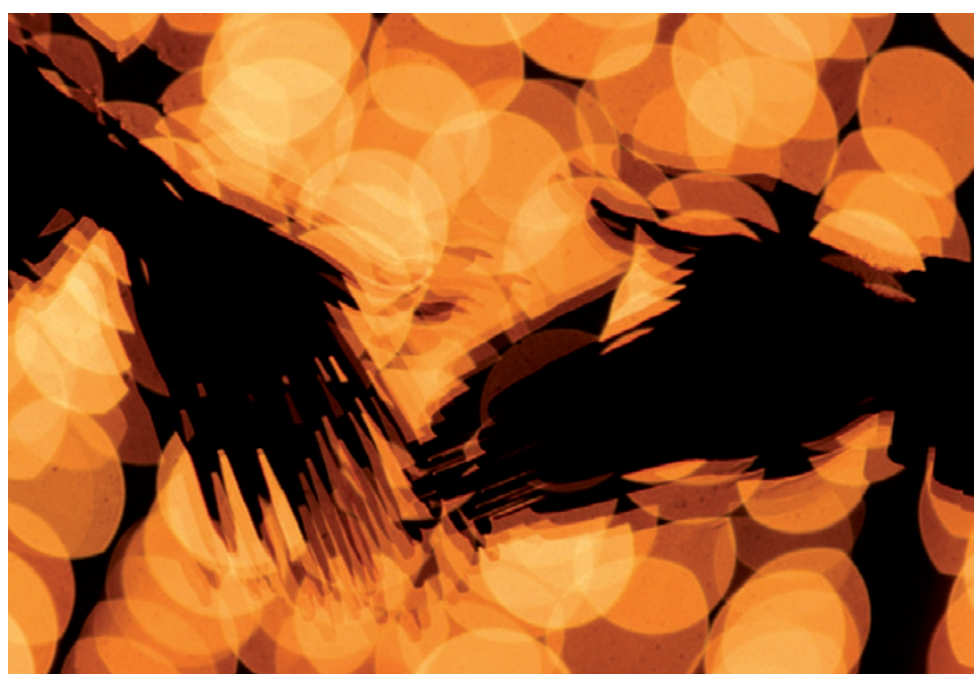

mice (which harbour $\mathrm{T}_{\text {Reg }}$ cells that express diphtheria toxin receptor), which permitted them to ablate $\mathrm{T}_{\text {Reg }}$ cells in vivo by introducing diphtheria toxin. Surprisingly, $\mathrm{T}_{\mathrm{Reg}}$-cell ablation did not enhance sterilizing immunity (or viral clearance or protective immunity) as expected, but instead resulted in an accelerated fatal infection, suggesting that $\mathrm{T}_{\text {Reg }}$ cells enhance protective immunity to HSV infection.

The authors speculated that the ablation of $\mathrm{T}_{\text {Reg }}$ cells might have interfered with the production of indispensable antiviral cytokines by other immune cells. Indeed, interferon levels at the vaginal mucosa, the site of infection, were low in $\mathrm{T}_{\mathrm{Reg}}$-cell depleted mice, but, as these were actually augmented in the draining lymph nodes, could it be possible that the ablation of $\mathrm{T}_{\mathrm{Reg}}$ cells might be impairing trafficking of immune cells to the site of infection? Examination of effector immune-cell subsets confirmed that, at the early stage of infection, the reduced interferon production at the site of infection was associated with a delay in the arrival of natural killer cells, dendritic cells and $\mathrm{T}$ cells. They further showed that $\mathrm{T}_{\text {Reg }}$ cells balance the migration of immune effector cells from the site of immune induction to the site of infection by directly suppressing proinflammatory chemokine cues in the lymph nodes.

This unexpected finding of a role for $\mathrm{T}_{\mathrm{Reg}}$ cells in promoting protective immunity was not only specific to HSV infection as it could be extended to a systemic infection with lymphocytic choriomeningitis virus. Marta Tufet

ORIGINAL RESEARCH PAPER Lund, J. M. et al. Coordination of early protective immunity to viral infection by regulatory T cells. Science 24 April 2008 (doi:10.1126/science.1155209) 